IFUM 557/FT

\title{
Cohomology and Renormalization of BFYM Theory in three Dimensions
}

\author{
Alberto Accardi \\ Dipartimento di Fisica, Università di Milano \\ Via Celoria 1620133 Milano ITALY \\ Andrea Belli \\ Dipartimento di Fisica, Università di Milano \\ Via Celoria 1620133 Milano ITALY \\ Maurizio Martellini 1 \\ Dipartimento di Fisica, Università di Milano, \\ I.N.F.N. - Sezione di Milano, \\ Via Celoria 1620133 Milano ITALY \\ and \\ Landau Network at "Centro Volta", Como, ITALY \\ Mauro Zeni 2 \\ Dipartimento di Fisica, Università di Milano \\ and \\ I.N.F.N. - Sezione di Milano, \\ Via Celoria 1620133 Milano ITALY
}

\begin{abstract}
The first order formalism for 3D Yang-Mills theory is considered and two different formulations are introduced, in which the gauge theory appears to be a deformation of the topological BF theory. We perform the quantization and the algebraic analysis of cohomology and renormalization for both the models, which are found to be anomaly free. We discuss also their stability against radiative corrections, giving the full structure of possible counterterms, requiring an involved matricial renormalization of fields and sources.
\end{abstract}

\footnotetext{
${ }^{1}$ E-mail: martellini@vaxmi.mi.infn.it

${ }^{2}$ E-mail: zeni@vaxmi.mi.infn.it
} 


\section{Introduction}

The interplay among the topological and non perturbative properties of field theory is subject to increasing investigations in different areas of theoretical physics. In particular in gauge field theories the role of topologically non trivial configurations, e.g. instantons and monopoles, has been repeatedly conjectured to be related with the long range behaviour of the theory. The topological role of these configurations is better displayed in the computation of the intersection numbers associated to the vev's of non local observables supported on non trivial manifolds in the framework of topological field theories The topological theory may be either the twisted version of a suitable $N=2$ supersymmetric theory [1] or a topological theory of BF type [2, 3, 4].

The relation among topological and gauge field theories may be considered also in the usual case of bosonic Yang-Mills theory. Introducing the first-order formalism, the YangMills theory may be formally written as the deformation of the topological pure BF theory [5, 6, 7]. This formulation, named BFYM, suggests that new non local observables can be inherited by the gauge theory from the topological one [8] and indeed recently in terms of its enlarged field content an explicit realization of the 't Hooft algebra [9] has been given in the $4 \mathrm{D}$ case [0]. It is therefore important to quantize this theory and check its equivalence with the standard formulation of Yang-Mills. In particular the perturbative behaviour and renormalization properties are expected to agree with the usual case. In the $4 \mathrm{D}$ case the asymptotic free behaviour of the perturbative formulation of BFYM has been recently verified [6]. In this paper we address to the 3D case and study the renormalization properties of BFYM using cohomological tools. The extension of this analysis to the $4 \mathrm{D}$ case will be discussed elsewhere.

Two different first order formulations can be given, with a different symmetry and field content but with the same number of degrees of freedom, both corresponding to the standard Yang-Mills theory. Using algebraic analysis we consider the quantization and the perturbative formulation of these models, discuss the absence of anomalies and their stability against radiative corrections and the structure of the required counterterms. In particular, a matricial mixing among fields and external sources is introduced in order to produce the correct renormalization of the theory.

The plan of the paper is the following. In section 2 we introduce the classical model in both the formulations, named "gaussian" and "extended" BFYM, giving their field contents and BRST quantization; we discuss also the equivalence between the two models and of the models with the Yang-Mills theory. In section 3 the cohomological analysis of the gaussian model is performed after introducing a suitable Chern-Simons IR regulator, owing to the superrenormalizable character of 3D gauge theory. The gaussian model turns out to be stable and anomaly free. The matricial renormalization of fields and sources is described. In section 4 the same analysis is repeated for the extended model which again turns out to be stable and anomaly free. In the appendices we collect and discuss the propagators and the Feynman rules for both the models, the Ward identities for the two point functions and the notation and the conventions. 


\section{Classical model}

We consider the classical YM lagrangean in flat euclidean space-time written in the first order formalism:

$$
\mathcal{L}_{B F Y M}=i \operatorname{Tr}(B \wedge F)+g^{2} \operatorname{Tr} B^{2},
$$

with the notation $\operatorname{Tr} B^{2} \equiv \operatorname{Tr} B \wedge * B$; indeed integrating over the auxiliary field $B$ in the partition function we get $Z=e^{-\int \mathcal{L}_{Y M}}$ where

$$
\mathcal{L}_{Y M}=\frac{1}{4 g^{2}} \operatorname{Tr} F^{2} .
$$

This theory is invariant with respect to the gauge transformations, which reads in the infinitesimal form

$$
\begin{aligned}
& \delta A=-\mathrm{d}_{A} c, \\
& \delta B=i[B, c] .
\end{aligned}
$$

We note that in the limit of zero coupling constant we recover, at least formally, the pure

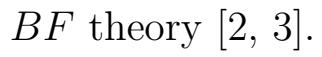

We have two ways to quantize the theory. In the first one we consider $\mathcal{L}_{0}=\operatorname{Tr}(B \wedge$ $\left.\mathrm{d} A+g^{2} B^{2}\right)$ as the free lagrangean and we have to fix the gauge symmetry: this will be done in section 2.1. In the second one we extend the symmetry group to that of the term $\mathcal{L}_{0}=\operatorname{Tr} B \wedge \mathrm{d} A$ which is larger than the one of the whole lagrangean (2.1), as it includes one more symmetry which we call "topological". To deal with this problem we will enlarge the field content of the theory introducing a zero form $\eta$ which will allow to extend the new symmetry to the whole lagrangean. This will be dealt with in section 2.2

\subsection{Gaussian model}

The BRST quantization of (2.1) is accomplished in the usual way by introducing the couple of ghost and antighost $(c, \bar{c})$ and the auxiliary field $b$ and by defining the nihilpotent BRST transformations as

$$
\begin{aligned}
s A & =-\mathrm{d}_{A} c, \\
s c & =-\frac{i}{2}[c, c], \\
s \bar{c} & =b, \\
s b & =0, \\
s B & =i[B, c] .
\end{aligned}
$$

Then we define the gauge-fixing lagrangean, choosing the covariant linear gauge,

$$
\mathcal{L}_{g f}=s\left(\bar{c} \wedge * \mathrm{~d}^{\dagger} A+\frac{\alpha}{2} b \wedge * \bar{c}\right) ;
$$

eventually the gauge-fixed lagrangean is

$$
\mathcal{L}=i B \wedge F+g^{2} B^{2}+\bar{c} \wedge * \mathrm{~d}^{\dagger} \mathrm{d}_{A} c+b \wedge * \mathrm{~d}^{\dagger} A+\frac{\alpha}{2} b^{2} .
$$


If we invert the quadratic operator appearing in the action, we get an off-diagonal structure for the propagators matrix which is reported in appendix A with the Feynman rules of the theory. Note that only a trilinear interaction vertex $B A A$ appears in the classical BFYM lagrangean and the off-diagonal propagators are actualy those that recover the non linear self interactions of Yang-Mills theory.

\subsection{Deformation of the pure BF theory}

In this section we aim to construct a theory equivalent to the gaussian formulation of the BFYM, but enjoying also the topological symmetry of the pure BF theory. This will be the starting point for the interpretation of the BFYM as a deformation of the pure BF theory.

Indeed the $B \wedge F$ term in (2.1) is invariant under the mapping $B \rightarrow B-\mathrm{d}_{A} \phi$, and this is precisely the symmetry which in the pure BF case spoils the local degrees of freedom. In the gaussian formulation the topological symmetry was broken by the $B^{2}$ term, so that we are naturally led to the introduction of a scalar field $\eta$ that can absorb the breaking of the symmetry.

We start using a Faddeev-Popov argument; consider the partition function of the gauge-fixed action of the previous section, where we write the functional measure $\mathcal{D} A$ and the gauge-fixing as $[\mathcal{D} A]$

$$
Z_{B F Y M}=\int[\mathcal{D} A] \mathcal{D} B e^{-S_{B F Y M}} .
$$

Then we define a Faddeev-Popov determinant $\Delta_{\mathcal{F}}$ satisfying

$$
1=\Delta_{\mathcal{F}}[B] \int \mathcal{D} \eta \delta\left(\mathcal{F}\left[B+\mathrm{d}_{A} \eta\right]\right),
$$

where $\eta$ is a zero form with values in the Lie algebra of the gauge group and $\mathcal{F}$ is a local functional with only the dependence on $B$ explicited; we note that we are considering infinitesimal transformation of the field $B$ in the direction $\mathrm{d}_{A} \eta$. Since the functional measure $\mathcal{D} \eta$ is invariant with respect to the translations of the field $\eta$, the determinant satisfies

$$
\Delta_{\mathcal{F}}[B]=\Delta_{\mathcal{F}}\left[B+\mathrm{d}_{A} \eta\right] .
$$

Inserting (2.8) into $Z_{B F}$ we obtain

$$
Z_{B F Y M}=\int[\mathcal{D} A] \mathcal{D} B \mathcal{D} \eta \Delta_{\mathcal{F}}[B] \delta\left(\mathcal{F}\left[B+\mathrm{d}_{A} \eta\right]\right) e^{-S_{B F}} .
$$

If we change variables

$$
\begin{aligned}
& B \longrightarrow B+\mathrm{d}_{A} \eta, \\
& \mathcal{D} B \longrightarrow \mathcal{D} B,
\end{aligned}
$$

and use the Bianchi identity we finally get

$$
Z_{B F Y M}=\int[\mathcal{D} A] \mathcal{D} B \mathcal{D} \eta \Delta_{\mathcal{F}}[B] \delta(\mathcal{F}[B]) e^{-\int d^{3} x\left(i \operatorname{Tr}(B \wedge F)+g^{2} \operatorname{Tr}\left(B+\mathrm{d}_{A} \eta\right)^{2}\right)},
$$


where we can see that the parameter of the transformation (2.11) has become dynamical. Therefore the lagrangean in the extended formulation becomes

$$
\mathcal{L}_{B F Y M \eta}=i \operatorname{Tr}(B \wedge F)+g^{2} \operatorname{Tr}\left(B+\mathrm{d}_{A} \eta\right)^{2},
$$

which is invariant with respect to the gauge symmetry

$$
\begin{aligned}
\delta_{g} A & =-\mathrm{d}_{A} c, \\
\delta_{g} B & =i[B, c], \\
\delta_{g} \eta & =i[\eta, c],
\end{aligned}
$$

and the topological symmetry

$$
\begin{aligned}
\delta_{\text {top }} A & =0, \\
\delta_{\text {top }} B & =-\mathrm{d}_{A} \phi, \\
\delta_{\text {top }} \eta & =\phi,
\end{aligned}
$$

which act on $A$ and $B$ as the gauge and topological symmetries of the pure BF theory. The field equations of the extended lagrangean are

$$
\begin{array}{r}
i * \mathrm{~d}_{A} B-2 i g^{2}\left[\eta, B+\mathrm{d}_{A} \eta\right]=0 \\
i * F+2 g^{2}\left(B+\mathrm{d}_{A} \eta\right)=0 \\
\mathrm{~d}_{A}\left(B+\mathrm{d}_{A} \eta\right)=0,
\end{array}
$$

and again the standard Yang-Mills theory is recovered by substituting them back in the lagrangean or by gaussian integration

Choosing the gauge fixing condition $\mathcal{G}=\mathrm{d}^{\dagger} A=0$ for the gauge symmetry and $\mathcal{F}=$ $\mathrm{d}^{\dagger} B=0$ for the topological symmetry

$$
\mathcal{L}_{g f}=s\left(\bar{c} \wedge * \mathrm{~d}^{\dagger} A+\frac{\alpha}{2} b \wedge * \bar{c}+\bar{\phi} \wedge * \mathrm{~d}^{\dagger} B+\frac{\beta}{2} h \wedge * \bar{\phi}\right),
$$

recovering the BRST invariance for the full theory under the nihilpotent BRST transformations $s$ defined as follows:

$$
\begin{aligned}
s A & =-\mathrm{d}_{A} c, \\
s c & =-\frac{i}{2}[c, c], \\
s \bar{c} & =b, \\
s b & =0, \\
s B & =i[B, c]-\mathrm{d}_{A} \phi, \\
s \phi & =-i[\phi, c], \\
s \bar{\phi} & =h, \\
s h & =0, \\
s \eta & =i[\eta, c]+\phi,
\end{aligned}
$$

\footnotetext{
${ }^{3} \mathrm{~A}$ more natural choice would have been $\mathrm{d}_{A}^{\dagger} B=0$ which excludes the transverse B-fields completely, but we preferred $\mathrm{d}^{\dagger} B=0$ because it leads to a more direct comparison with the works on pure BF theory [10
} 
with

$$
s^{2}=0 .
$$

The gauge fixed action can be interpreted as a deformation of the quantized pure BF theory

$$
Z_{B F Y M}=\int \mathcal{D} A \mathcal{D} B \mathcal{D} \eta \mathcal{D} c \mathcal{D} \bar{c} \mathcal{D} b \mathcal{D} \phi \mathcal{D} \bar{\phi} \mathcal{D} h e^{-S_{B F q}-g^{2} S_{\text {def }}},
$$

where

$$
\begin{aligned}
& S_{B F q}= \int\left\{i B \wedge F+\bar{c} \wedge * \mathrm{~d}^{\dagger} \mathrm{d}_{A} c+\bar{\phi} \wedge * \mathrm{~d}^{\dagger} \mathrm{d}_{A} \phi-i \bar{\phi} \wedge * \mathrm{~d}^{\dagger}[B, c]\right. \\
&\left.+b \wedge * \mathrm{~d}^{\dagger} A+h \wedge * \mathrm{~d}^{\dagger} B+\frac{\alpha}{2} b^{2}+\frac{\beta}{2} h^{2}\right\}, \\
& S_{\text {def }}=\int\left(B+\mathrm{d}_{A} \eta\right)^{2},
\end{aligned}
$$

and $S_{B F q}$ is the action of the topological pure BF theory quantized in in the same gauge [10]. We will call this formulation "extended BFYM". The extended formulation becomes even more interesting in the 4D case, where the topological symmetry is reducible presenting therefore a larger field contents [7].

Like in the gaussian model, from the Feynman rules we obtain an off-diagonal structure in the propagator matrix whose explicit form is reported in appendix 5 .

Finally we will count the degrees of freedom of the deformation of the BF, and check that it has just 1 bosonic degree of freedom as the gaussian formulation has. This is done by analizing the free part of the partition function and counting the number of bosonic and fermionic determinants [3]. Define

$$
\begin{aligned}
& \Delta_{0}=\mathrm{d}^{\dagger} \mathrm{d}: \Lambda^{0} \longrightarrow \Lambda^{0}, \\
& \Delta_{1}=\mathrm{d}^{\dagger} \mathrm{d}+\mathrm{dd}^{\dagger}: \Lambda^{1} \longrightarrow \Lambda^{1},
\end{aligned}
$$

where $\Lambda^{n}$ is the space of the Lie algebra valued $n$-forms. The integration on the ghosts $(c, \bar{c})$ and $(\phi, \bar{\phi})$ yields $\left(\operatorname{det} \Delta_{0}\right)^{2}$, and that on $\eta$ gives $\left(\operatorname{det} \Delta_{0}\right)^{-\frac{1}{2}}$. The integration on the remaining bosonic fields requires more care; in fact for a non-diagonal quadratic selfadjoint operator $K$ the determinant is defined as $\operatorname{det} K=\left(\operatorname{det}\left(K^{\dagger} K\right)\right)^{\frac{1}{2}}$ [11], so that

$$
\int e^{-(\rho, K \rho)}=(\operatorname{det} K)^{-\frac{1}{2}}=\left(\operatorname{det}\left(K^{\dagger} K\right)\right)^{\frac{1}{4}} .
$$

Then the integration on the fields $A, B, b$ and $h$ yields

$$
\left(\operatorname{det} \Delta_{1}\right)^{-\frac{1}{2}}\left(\operatorname{det} \Delta_{0}\right)^{-\frac{1}{2}} \text {. }
$$

In conclusion the free partition function is

$$
Z_{\eta 0}=\left(\operatorname{det} \Delta_{1}\right)^{-\frac{1}{2}}\left(\operatorname{det} \Delta_{0}\right)^{-\frac{1}{2}}\left(\operatorname{det} \Delta_{0}\right)^{2}\left(\operatorname{det} \Delta_{0}\right)^{-\frac{1}{2}} .
$$

Since the operator $\Delta_{1}$ is seen as $\Delta_{0}$ acting on three copies of $\Lambda^{0}$, when concerning the degree of freedom count, eventually we are left with 1 bosonic degree of freedom, as it happens in YM and in the gaussian formulation of BFYM. 


\subsubsection{Energy-momentum tensor}

The theory described by (2.17) is not a topological one although it has the simmetry content of the pure BF theory. This can be demonstrated showing that the energy momentum tensor is not BRST-exact; indeed this is the condition which encodes the topological nature of a theory [12]. The tensor can be decomposed into three pieces corresponding to the pure $\mathrm{BF}$, gauge-fixing and deformation lagrangean:

$$
T_{\mu \nu}=T_{\mu \nu}^{B F}+T_{\mu \nu}^{g f}+T_{\mu \nu}^{d e f}
$$

where $T_{\mu \nu}^{B F}=0$, owing to the fact the the pure BF lagrangean is metric independent.

$T_{\mu \nu}^{g f}=\left\{Q, \frac{2}{\sqrt{g}} \frac{\delta \Psi}{\delta g^{\mu \nu}}\right\}$ where $\Psi$ is the gauge fermion of the BRST quantization procedure and $Q$ is the BRST charge . Then, an explicit calculation shows that

$$
T_{\mu \nu}^{d e f}=\frac{1}{2} g^{2}\left(B_{\lambda}^{a}+D_{\lambda} \eta^{a}\right)\left[\partial_{\mu} \eta^{a} \delta_{\nu \lambda}+\partial_{\nu} \eta^{a} \delta_{\mu \lambda}-\delta_{\mu \nu}\left(B_{\lambda}^{a}+D_{\lambda} \eta^{a}\right)\right]
$$

It is now easy to show that since in this relation there appear terms in $\eta$ but no terms in $\phi$ the tensor $T_{\mu \nu}^{\text {def }}$ cannot be BRST-exact. Indeed $\frac{\delta}{\delta \eta}(s \varphi)=0 \quad \forall \varphi=(A, B, \ldots$.$) so that \eta$ can appear in a BRST variation only as

$$
s\left(\eta M_{\mu \nu}[\varphi]+N_{\mu \nu}[\varphi \neq \eta]\right)=\eta s\left(M_{\mu \nu}[\varphi]\right)+(\phi+i[\eta, c]) M_{\mu \nu}[\varphi]+s N_{\mu \nu}[\varphi \neq \eta] \neq T_{\mu \nu}^{d e f}
$$

for any local functional $M_{\mu \nu}$ and $N_{\mu \nu}$. Therefore the theory is not topological; the local degrees of freedom which are spoiled by the gauge fixing of the added topological symmetry are recovered by the introduction of the field $\eta$.

\section{Cohomology and renormalization of the gaussian model}

We now consider the perturbative behaviour of the theory. The gaussian model is a superrenormalizable theory and due to the masslessness of its fields it presents IR divergences of ever increasing order in perturbation theory.

These divergences appear somewhat artificial. For example it can be shown [13, 14] that they appear because we are forcing a Taylor series in $g^{2} / p$ while the functions we are calculating are non analitical; indeed an appropriate resummation of the perturbative series shows that we should also take into account powers of the logarithms of $g^{2} / p$. Some other "cures" to these divergences have been investigated but in any case they are of a non perturbative nature.

A way to save the perturbation theory is to introduce a mass term for at least some of the fields. In the context of the gauge theories this is usually done by a Higgs mechanism, but in three dimensions another method is available: the addition of a Chern-Simons term to the lagrangean [15, 16]. Then all the propagators between the $A$ and $B$ field acquire a mass and in the Landau gauge the theory is safe from IR divergences. The zero mass limit, which formally recovers the massless theory, is argued to be smooth 
for resummed quantities and moreover the observables should be mass independent 14. The IR problem arises in our analysis when we want to study the quantum extendibility of the classical constraints of our theory. Indeed the main tool for this analysis is the Quantum Action Principle (QAP) [17, 18, which is valid for UV and IR renormalizable theories, and BRST invariance [19]. The addition of the Chern-Simons term makes the theory IR renormalizable by power-counting in the Landau gauge; for the YMCS case the renormalizability is explicitly shown in 20 by perturbative calculations to one-loop order and by a cancellation theorem valid to all orders, and in [21] the calculations are performed to two-loops. The Chern-Simons term has another interesting feature: it does not change either the algebraic structure or the form of the operators entering in the algebraic analysis (in particular the S-T operator is the same as in the massless theory). For these reasons we shall adopt this IR regularization and we shall restrict our analysis to the Landau gauge.

\subsection{Classical analysis}

The classical (or tree level) lagrangean of the regularized theory is

$$
\begin{aligned}
\mathcal{L}= & \mathcal{L}_{B F Y M}+i m \mathcal{L}_{\mathrm{CS}}+\mathcal{L}_{g f}+\mathcal{L}_{\text {sources }}= \\
= & i B \wedge F+B^{2}+i m\left(A \wedge \mathrm{d} A+\frac{2}{3} g A \wedge A \wedge A\right)+ \\
& +\left(\bar{c} \wedge * \mathrm{~d}^{\dagger} \mathrm{d}_{A} c+b \wedge * \mathrm{~d}^{\dagger} A\right)+\left(\Omega_{A} \wedge * s(A)+\Omega_{B} \wedge * s(B)+\Omega_{c} \wedge * s(c)\right)
\end{aligned}
$$

where we added the external sources coupled to the non-linear BRST variations of the fields and rescaled the fields as $A \rightarrow g A, B \rightarrow B / g$, in order that their UV dimensions match the physical dimensions, The dimensions, ghost-number, Grassmann and spacetime inversion parity of the fields are shown in table 1.

\begin{tabular}{|l||c|c|c|c|c|c|c|c|}
\hline & $A$ & $B$ & $c$ & $\bar{c}$ & $b$ & $\Omega_{A}$ & $\Omega_{B}$ & $\Omega_{c}$ \\
\hline \hline UV dimension & $\frac{1}{2}$ & $\frac{3}{2}$ & 0 & 1 & $\frac{3}{2}$ & 2 & $\frac{3}{2}$ & 3 \\
\hline IR dimensions & 1 & $\frac{3}{2}$ & 0 & 1 & $\frac{3}{2}$ & 2 & $\frac{3}{2}$ & 3 \\
\hline Ghost number & 0 & 0 & 1 & -1 & 0 & -1 & -1 & -2 \\
\hline Grassmann parity & + & + & - & - & + & - & - & + \\
\hline Space-time parity & - & + & + & + & + & - & + & + \\
\hline
\end{tabular}

Table 1: dimensions, ghost-number and parity of the fields

Note that under the simultaneous reflection of all the coordinate axis $\mathcal{L}_{\mathrm{CS}} \longrightarrow-\mathcal{L}_{\mathrm{CS}}$, so that the IR regularized theory is parity-breaking.

The classical action $\Sigma=\int \mathcal{L}$ is characterized by the gauge-fixing condition

$$
\frac{\delta \Sigma}{\delta b}=\partial_{\mu} A_{\mu}
$$


and by the Slavnov-Taylor identity, which is a consequence of the BRST invariance,

$$
\mathcal{S}(\Sigma)=0
$$

where

$$
\mathcal{S}(\Sigma)=\int \mathrm{d}^{3} x\left(\frac{\delta \Sigma}{\delta A_{\mu}^{a}} \frac{\delta \Sigma}{\delta \Omega_{A \mu}^{a}}+\frac{\delta \Sigma}{\delta B_{\mu}^{a}} \frac{\delta \Sigma}{\delta \Omega_{B \mu}^{a}}+b^{a} \frac{\delta \Sigma}{\delta \bar{c}^{a}}+\frac{\delta \Sigma}{\delta c^{a}} \frac{\delta \Sigma}{\delta \Omega_{c}^{a}}\right) .
$$

We define the linearized Slavnov operator as

$$
\begin{aligned}
& B_{\Sigma}=\int \mathrm{d}^{3} x\left(\frac{\delta \Sigma}{\delta A_{\mu}^{a}} \frac{\delta}{\delta \Omega_{A \mu}^{a}}+\frac{\delta \Sigma}{\delta \Omega_{A \mu}^{a}} \frac{\delta}{\delta A_{\mu}^{a}}+\frac{\delta \Sigma}{\delta B_{\mu}^{a}} \frac{\delta}{\delta \Omega_{B \mu}^{a}}+\frac{\delta \Sigma}{\delta \Omega_{B \mu}^{a}} \frac{\delta}{\delta B_{\mu}^{a}}+\right. \\
&\left.+b^{a} \frac{\delta}{\delta \bar{c}^{a}}+\frac{\delta \Sigma}{\delta c^{a}} \frac{\delta}{\delta \Omega_{c}^{a}}+\frac{\delta \Sigma}{\delta \Omega_{c}^{a}} \frac{\delta}{\delta c^{a}}\right)
\end{aligned}
$$

whose dimensions are $d_{U V}=\frac{7}{2}$ and $d_{I R}=3$; from (3.5) follows the nihilpotency of this operator,

$$
B_{\Sigma} B_{\Sigma}=0
$$

Moreover by commuting (3.4) and (3.5) we obtain the antighost equation of motion

$$
\begin{aligned}
& \overline{\mathcal{G}}^{a} \Sigma=0 \\
& \overline{\mathcal{G}}^{a}=\frac{\delta}{\delta \bar{c}^{a}}+\partial_{\mu} \frac{\delta}{\delta \Omega_{A \mu}},
\end{aligned}
$$

whose consequence is that the source $\Omega$ and the antighost enter in the action only through the combination:

$$
\widehat{\Omega}=\Omega+\partial_{\mu} \bar{c} .
$$

With respect to this new variable we define the reduced action

$$
\widehat{\Sigma}\left[A, B, c, \widehat{\Omega}_{A}, \Omega_{B}, \Omega_{c}\right]=\Sigma\left[A, B, c, \bar{c}, b, \Omega_{A}, \Omega_{B}, \Omega_{c}\right]-b \wedge * \mathrm{~d}^{\dagger} A
$$

which clearly satisfies

$$
\frac{\delta \widehat{\Sigma}}{\delta b}=0
$$

The S-T operator becomes

$\widehat{B}_{\widehat{\Sigma}}=\int \mathrm{d}^{3} x\left(\frac{\delta \widehat{\Sigma}}{\delta A_{\mu}^{a}} \frac{\delta}{\delta \widehat{\Omega}_{A_{\mu}^{a}}}+\frac{\delta \widehat{\Sigma}}{\delta \widehat{\Omega}_{A_{\mu}}^{a}} \frac{\delta}{\delta A_{\mu}^{a}}+\frac{\delta \widehat{\Sigma}}{\delta B_{\mu}^{a}} \frac{\delta}{\delta \Omega_{B \mu}^{a}}+\frac{\delta \widehat{\Sigma}}{\delta \Omega_{B \mu}^{a}} \frac{\delta}{\delta B_{\mu}^{a}}+\frac{\delta \widehat{\Sigma}}{\delta c^{a}} \frac{\delta}{\delta \Omega_{c}^{a}}+\frac{\delta \widehat{\Sigma}}{\delta \Omega_{c}^{a}} \frac{\delta}{\delta c^{a}}\right)$,

and the S.T. identity (3.5) is rewritten as

$$
\widehat{B}_{\widehat{\Sigma}} \widehat{\Sigma}=0
$$

Another constraint on $\widehat{\Sigma}$ is given in the Landau gauge by the ghost equation [22] which reads

$$
\mathcal{G}^{a} \Sigma=\Delta_{(g)}^{a}
$$


where

$$
\begin{aligned}
\mathcal{G}^{a} & =\int \mathrm{d}^{3} x\left(\frac{\delta}{\delta c^{a}}+f^{a b c} \bar{c}^{b} \frac{\delta}{\delta b^{c}}\right), \\
\Delta_{(g)}^{a} & =\int \mathrm{d}^{3} x f^{a b c}\left(\Omega_{A \mu}^{b} A_{\mu}^{c}+\Omega_{B \mu}^{b} B_{\mu}^{c}-\Omega_{c}^{b} c^{c}\right) .
\end{aligned}
$$

The action is invariant also with respect to the rigid gauge transformations of parameter $\omega$

$$
\delta_{\text {rig }} \varphi=[\omega, \varphi] \quad \varphi=A, B, c, \bar{c}, b, \Omega_{A}, \Omega_{B}, \Omega_{c},
$$

whose Ward identity is

$$
W^{r i g} \Sigma=\int \mathrm{d}^{3} x \sum_{\varphi}\left[\varphi, \frac{\delta \Sigma}{\delta \varphi}\right]_{ \pm}=0,
$$

where we used commutators for the bosonic fields and anticommutators for the fermionic one. In the Landau gauge the Ward identity (3.18) may also be derived by commuting (3.14) with the S.T. identity.

In summary, the classical action is characterized by the following constraints

$$
\begin{aligned}
& \widehat{B_{\widehat{\Sigma}} \widehat{\Sigma}}=0, \\
& \frac{\delta \widehat{\Sigma}}{\delta b^{a}}=0, \\
& \overline{\mathcal{G}}^{a} \widehat{\Sigma}=\frac{\delta}{\delta \bar{c}} \widehat{\Sigma}=0, \\
& \mathcal{G}^{a} \widehat{\Sigma}=\int \mathrm{d}^{3} x \frac{\delta}{\delta c} \widehat{\Sigma}=0, \\
& W^{r i g} \widehat{\Sigma}=\int \mathrm{d}^{3} x\left[\varphi, \frac{\delta \widehat{\Sigma}}{\delta \varphi}\right]_{ \pm}=0 .
\end{aligned}
$$

The action of the linearized Slavnov-Taylor operator on the fields and on the sources is

$$
\begin{aligned}
\widehat{B}_{\widehat{\Sigma}} \varphi & =\frac{\delta \widehat{\Sigma}}{\delta \varphi}=s \varphi \quad \text { for } \varphi=A, B, c, \\
\widehat{B}_{\widehat{\Sigma}} \widehat{\Omega}_{A} & =\frac{\delta \widehat{\Sigma}}{\delta A}=i * \mathrm{~d}_{A} B+i m * F+i g\left\{\widehat{\Omega}_{A}, c\right\} \\
\widehat{B}_{\widehat{\Sigma}} \Omega_{B} & =\frac{\delta \widehat{\Sigma}}{\delta B}=i * F+2 B+i g\left\{\widehat{\Omega}_{B}, c\right\} \\
\widehat{B}_{\widehat{\Sigma}} \Omega_{c} & =\frac{\delta \widehat{\Sigma}}{\delta c}=\mathrm{d}_{A}^{\dagger} \widehat{\Omega}_{A}+i g *\left[\Omega_{B}, * B\right]+i g\left[\Omega_{c}, c\right] .
\end{aligned}
$$

\subsection{Renormalization of the theory}

In this section we will study the perturbative extension of the relations (3.19-3.23) and the stability of the theory under quantum corrections, i.e. the search of the more general invariant counterterms. The latter and the problem of the gauge anomaly will be translated into a problem of local cohomology for the operator $\widehat{B}_{\widehat{\Sigma}}$ to be solved in an appropriate space of local field functionals with fixed dimensions and ghost-number. In particular, being interested in the action, we can discard total derivatives so that we will be concerned with a problem of cohomology modulo d, which will be dealt with by considering an appropriate system of descent equations. 


\subsubsection{Anomaly}

It is easy to show that the conditions 3.203 .23 ) can be extended to all orders of perturbation by the introduction of non-invariant counterterms in the classical action [18], i.e. we can define a quantum vertex functional $\Gamma$ satisfying

$$
\begin{aligned}
& \frac{\delta \Gamma}{\delta b^{a}}=\partial_{\mu} A_{\mu}^{a}, \\
& \overline{\mathcal{G}}^{a} \Gamma=0, \\
& \mathcal{G}^{a} \Gamma=\Delta_{(g)}^{a}, \\
& \mathcal{H}_{r i g}^{a} \Gamma=0 .
\end{aligned}
$$

As a consequence we can decompose it as

$$
\Gamma\left[A, B, c, \bar{c}, b, \Omega_{A}, \Omega_{B}, \Omega_{c}\right]=\widehat{\Gamma}\left[A, B, c, \widehat{\Omega}_{A}, \Omega_{B}, \Omega_{c}\right]+\operatorname{Tr} \int \mathrm{d}^{3} \mathrm{x}\left(\mathrm{b} \wedge * \mathrm{~d}^{\dagger} \mathrm{A}\right) .
$$

We want now to study the gauge anomaly. Therefore we start by writing a broken S-T identity

$$
\widehat{B}_{\widehat{\Gamma}} \widehat{\Gamma}=\hbar^{n} \Delta_{0}^{1}+O\left(\hbar^{n+1}\right)
$$

where the break is a local field functional of ghost-number 1 and form degree 0 assumed to appear at the order $n$, and is constrained by the QAP to have UV dimensions less than $\frac{7}{2}$ and IR dimensions greater than 3. Thank to the commutation properties of the operators appearing in 3.253 .28 with the Slavnov-Taylor operator, the break must also to satisfy the following constraints:

$$
\begin{aligned}
\frac{\delta}{\delta b^{a}} \Delta & =\frac{\delta}{\delta h^{a}} \Delta=0 \\
\overline{\mathcal{G}}^{a} \Delta & =\overline{\mathcal{F}}^{a} \Delta=0 \\
\mathcal{G}^{a} \Delta & =0 \\
\mathcal{F}^{a} \Delta & =0 \\
\mathcal{H}_{\text {rig }}^{a} \Delta & =0 \\
\mathcal{N}_{\text {rig }}^{a} \Delta & =0 .
\end{aligned}
$$

The identity $\widehat{B}_{\gamma} \widehat{B}_{\gamma} \gamma=0, \forall \gamma$, along with the fact that $\widehat{B}_{\widehat{\Gamma}}=\widehat{B}_{\widehat{\Sigma}}+O(\hbar)$, implies that

$$
\widehat{B}_{\widehat{\Sigma}} \Delta_{0}^{1} \equiv \widehat{B}_{\widehat{\Sigma}} \int \Omega_{3}^{1}=0
$$

whose solution is to be found in the subspace satisfying the constraints (3.25-3.28). In general, the solution will be of the form

$$
\Omega_{3}^{1}=\widehat{\Omega}+\widehat{B}_{\widehat{\Sigma}} \widetilde{\Omega}_{3}^{0}+\mathrm{d} \bar{\Omega}_{2}^{1},
$$

where $\widehat{\Omega}$ represents the anomaly and $-\widetilde{\Omega}$ the non invariant counterterms to be added to the classical lagrangean at the order $n$.

It is possible to symplify the analysis by studying the cohomology of the linearized part $b_{0}$ of the operator $\widehat{B}$. Following general lines it can be demonstrated that the cohomology of $\widehat{B}$ is included in that of $b_{0}$ [18] and the following proposition holds 
Proposition 3.1 The local cohomology of $b_{0}$ is independent of the external sources and depends on

$$
\begin{aligned}
& \mathrm{d} A \text { and its derivatives, } \\
& c \quad \text { not derived, } \\
& B \text { and its derivatives. }
\end{aligned}
$$

The descent equations leading to the solution of (3.31) are

$$
\begin{aligned}
& b_{0} \Omega_{3}^{1}+\mathrm{d} \Omega_{2}^{2}=0, \\
& b_{0} \Omega_{2}^{2}+\mathrm{d} \Omega_{1}^{3}=0, \\
& b_{0} \Omega_{1}^{3}+\mathrm{d} \Omega_{0}^{4}=0, \\
& b_{0} \Omega_{0}^{4}=0,
\end{aligned}
$$

where $\Omega_{q}^{p}$ is a q-form of UV dimension bounded by q and ghost number p. The result is

$$
\Omega_{3}^{1}=b_{0} \Omega_{3}^{0}+\mathrm{d} \Omega_{2}^{1}+z_{1} \mathrm{~d} A(A c-c A)+z_{2} \mathrm{~d} B(A c-c A)+u_{1} \mathrm{~d} A * \mathrm{~d} A c+u_{2} B * B c
$$

The monomials in $u_{1}, u_{2}$ are null, indeed for example $\operatorname{Tr}(B * B c)=f^{a b c} B_{\mu}^{a} B_{\mu}^{b} c^{c}=0$. The monomials in $z_{1}$ and $z_{2}$ are trivial:

$$
\begin{aligned}
\mathrm{d} A(A c-c A) & =b_{0}\left[-\frac{1}{3} A^{3}\right]+\mathrm{d}\left[\frac{1}{2} \mathrm{~d}(A(A c-c A))\right] \\
\mathrm{d} B(A c-c A) & =\frac{1}{2} b_{0}\left[i * \mathrm{~d} A A^{2}-\mathrm{d} \Omega_{B}(A c-c A)\right]+\mathrm{d}\left[\Omega_{B} \mathrm{~d} c^{2}-i * \mathrm{~d} A(A c-c A)\right] .
\end{aligned}
$$

Therefore the cohomology of $b_{0}$ has no nontrivial terms in this sector. Then also the cohomology of $\widehat{B}_{\widehat{\Sigma}}$ is trivial and we conclude that the Slavnov-Taylor condition is not anomalous.

\subsubsection{Stability}

In this section we will search for the most general invariant counterterms, i.e. local field functionals with dimensions $d_{U V} \leq 3, d_{I R} \geq 3$ and ghost-number 0 which are invariant with respect to all the symmetries of the theory. In particular we will study the condition

$$
\widehat{B}_{\widehat{\Sigma}} L \equiv \widehat{B}_{\widehat{\Sigma}} \int \Omega_{3}^{0}=0
$$

in the local functional space constrained by the conditions (3.25, 3.26, 3.28) and the ghost equation

$$
\mathcal{G}^{a} L=0 \text {. }
$$

This means that we consider $L$ to be dependent on $\widehat{\Omega}$ and independent of $b$ and $\bar{c}$. The cohomology of the operator $b_{0}$ in this space is given by proposition 3.1.

\footnotetext{
${ }^{4}$ Traces and wedge products are always understood.
} 
The descent equations related to this problem are

$$
\begin{aligned}
& b_{0} \Omega_{3}^{0}+\mathrm{d} \Omega_{2}^{1}=0, \\
& b_{0} \Omega_{2}^{1}+\mathrm{d} \Omega_{1}^{2}=0, \\
& b_{0} \Omega_{1}^{2}+\mathrm{d} \Omega_{0}^{3}=0, \\
& b_{0} \Omega_{0}^{3}=0,
\end{aligned}
$$

and the solution is

$$
\Omega_{3}^{0}=b_{0} \widehat{\Omega}_{3}^{-1}+\mathrm{d} \widehat{\Omega}_{2}^{0}+i z_{1} B \mathrm{~d} A+z_{2} B * B+z_{3} \mathrm{~d} A * \mathrm{~d} A+i z_{4} m A \mathrm{~d} A .
$$

But the terms of coefficient $z_{1}$ and $z_{2}$ are equivalent to $\mathrm{d} A * \mathrm{~d} A$, indeed:

$$
\begin{aligned}
B \mathrm{~d} A & =-\frac{i}{2} \mathrm{~d} A * \mathrm{~d} A+b_{0}\left(\Omega_{B} \mathrm{~d} A\right) \\
B * B & =-\frac{i}{2} B \mathrm{~d} A+b_{0}\left(\Omega_{B} * B\right) .
\end{aligned}
$$

The extension to the cohomology of $\widehat{B}_{\widehat{\Sigma}}$ is straightforward:

$$
\widehat{B}_{\widehat{\Sigma}} \widetilde{\Omega}_{3}^{-1}+\mathrm{d} \widetilde{\Omega}_{2}^{0}+z_{3} F * F+i z_{4} g^{2} \mathcal{L}_{\mathrm{CS}} .
$$

We observe that this cohomology is equivalent to that of the YMCS theory.

The trivial part of the cohomology is given by the variation of $\widetilde{\Omega}_{3}^{-1}$, which is a local functional of dimension $d_{U V} \leq 3$ and $d_{I R} \geq 3$; therefore it is a superposition of the following monomials:

$$
\widehat{\Omega}_{A} * A \quad \Omega_{B} * B \quad \Omega_{c} * c \quad \Omega_{B} \mathrm{~d} A \quad \Omega_{B}[A, A] .
$$

The first three terms correspond to field renormalizations and the following two require that the renormalization allow a field mixing.

Let us introduce the following notation:

$$
N_{\varphi}=\int \varphi * \frac{\delta}{\delta \varphi} \quad ; \quad N_{\varphi \rightarrow \omega}=\int \omega * \frac{\delta}{\delta \varphi} .
$$

Then the trivial counterterms can be expressed as

$$
\begin{aligned}
& \widehat{B}_{\widehat{\Sigma}}\left(\widehat{\Omega}_{A} * A\right)=\left(N_{A}-N_{\widehat{\Omega}_{A}}\right) \widehat{\Sigma} \equiv \mathcal{N}_{A} \widehat{\Sigma}, \\
& \widehat{B}_{\widehat{\Sigma}}\left(\Omega_{B} * B\right)=\left(N_{B}-N_{\Omega_{B}}\right) \widehat{\Sigma} \equiv \mathcal{N}_{B} \widehat{\Sigma}, \\
& \widehat{B}_{\widehat{\Sigma}}\left(\Omega_{c} * c\right)=\left(N_{c}-N_{\Omega_{c}}\right) \widehat{\Sigma} \equiv \mathcal{N}_{c} \widehat{\Sigma}, \\
& \widehat{B}_{\widehat{\Sigma}}\left(\Omega_{B} \mathrm{~d} A\right)=\left(N_{B \rightarrow * \mathrm{~d} A}-N_{\widehat{\Omega}_{A} \rightarrow * \mathrm{~d} \Omega_{B}}\right) \widehat{\Sigma} \equiv \mathcal{N}_{\text {rot }}^{(1)} \widehat{\Sigma}, \\
& \widehat{B}_{\widehat{\Sigma}}\left(\Omega_{B}[A, A]\right)=\left(N_{B \rightarrow *[A, A]}-2 N_{\widehat{\Omega}_{A} \rightarrow *\left[A, \Omega_{B}\right]}\right) \widehat{\Sigma} \equiv \mathcal{N}_{\text {rot }}^{(2)} \widehat{\Sigma} .
\end{aligned}
$$

At last, the ghost equation 3.35 excludes the $\mathcal{N}_{c}$ counterterm.

It is now apparent that all the trivial counterterms are already present in the classical BFYM lagrangean or can be absorbed via an appropriate transformation of the fields and the parameters, which will be the subject of the next section. The absorption of the non-trivial counterterms will require more care. 


\subsection{Renormalization transformations}

In this section we will be concerned with the analysis of the transformations of the fields which permit to determine perturbatively the invariant counterterms needed to renormalize the theory. The appearance of terms not present in the classical lagrangean will force us to consider not only multiplicative transformations but to allow for a rotation in the space of the fields; the renormalization will be multiplicative only in a matricial sense.

For a better comprehension of what are the terms that contribute to the renormalization of the coupling constant we rescale the fields so that $g$ appears only in the terms $B^{2}$ and $F^{2}$.

To show in detail the absorbability of the counterterms, we proceed by induction and consider the counterterms absorbed till the order $n-1$. Then the results of section 3.2 .2 show that the counterterms enter in the action in the following manner

$$
\begin{aligned}
\Sigma= & \widehat{\Sigma}_{0}+\int\left(\hbar^{n} z_{3} \frac{1}{g^{2}} F_{0}^{2}+i \hbar^{n} z_{4} m \mathcal{L}_{\mathrm{CS} 0}+\right. \\
& \left.+\hbar^{n} a_{1} \mathcal{N}_{A} \widehat{\Sigma}_{0}+\hbar^{n} a_{2} \mathcal{N}_{B} \widehat{\Sigma}_{0}+\hbar^{n} a_{3} \mathcal{N}_{c} \widehat{\Sigma}_{0}+\hbar^{n} a_{4} \mathcal{N}_{\text {rot }}^{\prime} \widehat{\Sigma}_{0}+\hbar^{n} a_{5} \mathcal{N}_{\text {rot }}^{\prime \prime} \widehat{\Sigma}_{0}\right)
\end{aligned}
$$

where $\widehat{\Sigma}_{0}$ is the bare reduced classical action.

We separate the absorption procedure in three steps. At first, we absorb the $F^{2}$ term by a translation of the $B$-field; this step produces a counterterm of the type $B F$. Then we will absorb the $B F$ counterterm, by a rescaling of the $B$-field, of its source $\Omega_{B}$ and a renormalization of $g$; moreover we will absorb also the $\mathcal{L}_{\mathrm{CS}}$ counterterm by a mass renormalization. Note that only in these steps we have the physical renormalization of the two dimensionful parameters of the theory, $g$ and $m$. After these two steps we are left only with the trivial counterterms: we can therefore complete the procedure by a wave-function "matricial" renormalization.

We analize the three steps in detail:

1. Absorption of $\mathbf{F}^{\mathbf{2}}$. By a translation of the $B$-field we can extract from the $B^{2}$ term a monomial of the type $F^{2}$ (we cannot translate the $A$-field because we want its image to be a connection). However, this translation generates terms like $K_{2}\left[F_{2}, c_{2}\right]$ stemming from $K_{1} \wedge * s\left(B_{1}\right)$ but we can adsorb them by translating also the source $\widehat{\Omega}_{A}$ :

$$
\begin{aligned}
A_{0} & =A_{1} \\
B_{0} & =B_{1}+i \hbar^{n} \frac{1}{g^{2}} z_{3} * F_{1}, \\
c & =c_{1}, \\
\widehat{\Omega}_{A 0} & =\widehat{\Omega}_{A 1}-i \hbar^{n} z_{3} \frac{1}{g_{1}^{2}} * \mathrm{~d}_{A} \Omega_{B 1}, \\
\Omega_{B 0} & =\Omega_{B 1}, \\
\Omega_{c 0} & =\Omega_{c 1}, \\
g_{0} & =g_{1}, \\
m_{0} & =m_{1},
\end{aligned}
$$


where we have used the identity

$$
\int \Omega_{B} *[* F, c]=\int * \mathrm{~d}_{A} \Omega_{B} * \mathrm{~d}_{A} c
$$

We obtain therefore the following lagrangean:

$$
\begin{aligned}
\Sigma_{1}= & \widehat{\Sigma}\left[\varphi_{1}\right]+\int\left[i \hbar^{n} z_{3} B_{1} \wedge F_{1}+i \hbar^{n} z_{4} m \mathcal{L}_{\mathrm{CS} 1}+\right. \\
& \left.+\hbar^{n} a_{1} \mathcal{N}_{A} \widehat{\Sigma}_{1}+\hbar^{n} a_{2} \mathcal{N}_{B} \widehat{\Sigma}_{1}+\hbar^{n} a_{3} \mathcal{N}_{c} \widehat{\Sigma}_{1}+\hbar^{n} a_{4} \mathcal{N}_{\text {rot }}^{\prime} \widehat{\Sigma}_{1}-\frac{i}{2} \hbar^{n} a_{5} \mathcal{N}_{\text {rot }}^{\prime \prime} \widehat{\Sigma}_{1}\right]
\end{aligned}
$$

2. Absorption of $B F$ and $\mathcal{L}_{\mathrm{CS}}$. We can cancel the $B F$ counterterm in (3.46) by rescaling $B$. In so doing we produce also a $B^{2}$ term which can be absorbed by a coupling constant renormalization, and a $\Omega_{B}[B, c]$ term which can be cancelled by a rescaling of $\Omega_{B}:$

$$
\begin{aligned}
A_{1} & =A_{2}, \\
B_{1} & =B_{2}-\hbar^{n}\left(z_{3}\right) B_{2}, \\
c_{1} & =c_{2}, \\
\widehat{\Omega}_{A 1} & =\widehat{\Omega}_{A 2}, \\
\Omega_{B 1} & =\Omega_{B 2}+\hbar^{n}\left(z_{3}\right) \Omega_{B 2} \\
\Omega_{c 1} & =\Omega_{c 2}, \\
g_{1} & =g_{2}+\hbar^{n} z_{3} g_{2}, \\
m_{1} & =m_{2}-\hbar^{n} z_{4} m_{2} .
\end{aligned}
$$

We then obtain

$$
\begin{aligned}
\Sigma_{2}=\hat{\Sigma}\left[\varphi_{2}\right]+\int[ & \hbar^{n} a_{1} \mathcal{N}_{A} \widehat{\Sigma}_{2}+\hbar^{n} a_{2} \mathcal{N}_{B} \widehat{\Sigma}_{2}+\hbar^{n} a_{3} \mathcal{N}_{c} \widehat{\Sigma}_{2}+ \\
+ & \left.\hbar^{n} \frac{1}{g^{2}} a_{4} \mathcal{N}_{r o t}^{\prime} \widehat{\Sigma}_{2}-\frac{i}{2 g^{2}} \hbar^{n} a_{5} \mathcal{N}_{r o t}^{\prime \prime} \widehat{\Sigma}_{2}\right]
\end{aligned}
$$

Note that we could have chosen $B * B$ as the representative of the cohomology (3.39). In this case the previous steps would have reduced to the physical renormalizations only, but with our choice the relation with the renormalization of the YMCS theory is more direct, i.e. it is the $F^{2}$ term which gives the coupling constant renormalization in both cases. 
3. Wave function renormalization Finally, it is sufficient to rescale and rotate the fields and the sources to absorb the remaining counterterms:

$$
\begin{aligned}
A_{2} & =A_{R}-\hbar^{n} a_{1} A_{R}, \\
B_{2} & =B_{R}-\hbar^{n} a_{2} B_{R}-\hbar^{n} a_{4} * \mathrm{~d} A_{R}+i \hbar^{n} a_{5} * \frac{1}{2}\left[A_{R}, A_{R}\right], \\
c_{2} & =c_{R}, \\
\widehat{\Omega}_{A 2} & =\widehat{\Omega}_{A R}+\hbar^{n} a_{1} \widehat{\Omega}_{A R}+\hbar^{n} a_{4} * \mathrm{~d} \Omega_{B R}-i \hbar^{n} a_{5} *\left[A_{R}, \Omega_{B R}\right], \\
\Omega_{B 2} & =\Omega_{B R}+\hbar^{n} a_{2} \Omega_{B R}, \\
\Omega_{c 2} & =\Omega_{c R}, \\
g_{2} & =g_{R}, \\
m_{2} & =m_{R} .
\end{aligned}
$$

Finally

$$
\Sigma_{R}\left[\varphi_{R}\right]=\left.\widehat{\Sigma}[\varphi]\right|_{\varphi=\varphi_{R}}
$$

Complete renormalization transformations. Collecting the three steps in one transformation and collecting the fields and the sources in multiplets we can write the renormalization transformations in matrix notation:

$$
\left(\begin{array}{c}
\mathrm{d} A_{0} \\
-i\left[A_{0}, A_{0}\right] \\
B_{0} \\
c_{0}
\end{array}\right)=\left(\begin{array}{cccc}
1-\hbar^{n} a_{1} & 0 & 0 & 0 \\
0 & 1-\hbar^{n} a_{1} & 0 & 0 \\
1-\hbar^{n} \frac{1}{g^{2}}\left(i z_{3}+a_{4}\right) * & 1-\hbar^{n} \frac{1}{g^{2}}\left(i z_{3}+a_{5}\right) * & 1-\hbar^{n}\left(a_{2}+z_{3}\right) & 0 \\
0 & 0 & 0 & 0
\end{array}\right)\left(\begin{array}{c}
\mathrm{d} A_{R} \\
-\frac{i}{2}\left[A_{R}, A_{R}\right] \\
B_{R} \\
c_{R}
\end{array}\right)
$$

and

$$
\left(\begin{array}{c}
\widehat{\Omega}_{A 0} \\
\mathrm{~d} \Omega_{B 0} \\
-i\left[A_{0}, \Omega_{B 0}\right] \\
\Omega_{c 0}
\end{array}\right)=\left(\begin{array}{cccc}
1+\hbar^{n} a_{1} & 1+\hbar^{n} \frac{1}{g^{2}}\left(-i z_{3}+a_{4}\right) * & 1+\hbar^{n} \frac{1}{g^{2}}\left(-i z_{3}+a_{5}\right) * & 0 \\
0 & 1+\hbar^{n} a_{2} & 0 & 0 \\
0 & 0 & 1+\hbar^{n} a_{2} & 0 \\
0 & 0 & 0 & 0
\end{array}\right)\left(\begin{array}{c}
\widehat{\Omega}_{A R} \\
\mathrm{~d} \Omega_{B R} \\
-i\left[A_{R}, \Omega B_{R}\right] \\
\Omega_{c R}
\end{array}\right)
$$

to which we add the renormalization of the physical parameters:

$$
\begin{aligned}
g & =\left(1+\hbar^{n} z_{3}\right) g_{R}, \\
m & =\left(1-\hbar^{n} z_{4}\right) m_{R} .
\end{aligned}
$$

Whereas it is not apparent from the transformations (3.52) that the fields transform in a covariant way we observe that, e.g., $s B_{R}=\frac{\delta \Sigma_{R}}{\delta K_{R}}=i\left[B_{R}, c_{R}\right]_{R}$.

\section{Cohomology and renormalization of extended BFYM}

In this section we perform the cohomological analysis on the formulation of the extended BFYM model along the lines of the previous analysis. We quantize the model in the Landau gauge $\mathrm{d}^{\dagger} A=\mathrm{d}^{\dagger} B=0$, and IR regularize it with a Chern-Simons mass term. 


\subsection{Classical analysis}

The classical lagrangean with the addition of the CS term is

$$
\begin{aligned}
\mathcal{L}= & i B \wedge F+\left(B+\mathrm{d}_{A} \eta\right)^{2}+i m\left(A \wedge \mathrm{d} A+\frac{2}{3} g A \wedge A \wedge A\right)+ \\
& +\bar{c} \wedge * \mathrm{~d}^{\dagger} \mathrm{d}_{A} c+b \wedge * \mathrm{~d}^{\dagger} A+\bar{\phi} \wedge * \mathrm{~d}^{\dagger}\left(\mathrm{d}_{A} \phi+i g[B, c]\right)+h \wedge * \mathrm{~d}^{\dagger} B+ \\
& +\Omega_{A} \wedge * s(A)+\Omega_{B} \wedge * s(B)+\Omega_{\eta} \wedge * s(\eta)+\Omega_{c} \wedge * s(c)+\Omega_{\phi} \wedge * s(\phi) .
\end{aligned}
$$

The dimensions, ghost-numbers, Grassmann and space-time parity of the fields and of the external sources are shown in table 2. The classical action is characterized by the

\begin{tabular}{|l||c|c|c|c|c|c|c|c|c|c|c|c|c|c|}
\hline & $A$ & $B$ & $\eta$ & $c$ & $\bar{c}$ & $b$ & $\phi$ & $\bar{\phi}$ & $h$ & $\Omega_{A}$ & $\Omega_{B}$ & $\Omega_{c}$ & $\Omega_{\phi}$ & $\Omega_{\eta}$ \\
\hline \hline UV dimension & $\frac{1}{2}$ & $\frac{3}{2}$ & $\frac{1}{2}$ & 0 & 1 & $\frac{3}{2}$ & $\frac{1}{2}$ & $\frac{1}{2}$ & $\frac{1}{2}$ & 2 & $\frac{3}{2}$ & 3 & $\frac{5}{2}$ & $\frac{5}{2}$ \\
\hline IR dimension & 1 & $\frac{3}{2}$ & $\frac{1}{2}$ & 0 & 1 & $\frac{3}{2}$ & $\frac{1}{2}$ & $\frac{1}{2}$ & $\frac{1}{2}$ & 2 & $\frac{3}{2}$ & 3 & $\frac{5}{2}$ & $\frac{5}{2}$ \\
\hline Ghost number & 0 & 0 & 0 & 1 & -1 & 0 & 1 & -1 & 0 & -1 & -1 & -2 & -2 & -1 \\
\hline Grassm. parity & + & + & + & - & - & + & - & - & + & - & - & + & + & - \\
\hline Parity & - & + & - & + & + & + & - & - & - & - & + & + & - & - \\
\hline
\end{tabular}

Table 2: dimensions, ghost-number and Grassmann parity of the fields

following constraints:

$$
\begin{aligned}
& \frac{\delta \Sigma}{\delta b}=\partial_{\mu} A_{\mu}, \\
& \frac{\delta \Sigma}{\delta h}=\partial_{\mu} B_{\mu}, \\
& \overline{\mathcal{G}}^{a} \Sigma=0, \\
& \overline{\mathcal{F}}^{a} \Sigma=0, \\
& \mathcal{S}(\Sigma)=0,
\end{aligned}
$$

where

$$
\begin{aligned}
\mathcal{S}(\Sigma)=\int \mathrm{d}^{3} x\left(\frac{\delta \Sigma}{\delta A_{\mu}^{a}} \frac{\delta \Sigma}{\delta \Omega_{A \mu}{ }^{a}}+\frac{\delta \Sigma}{\delta B_{\mu}^{a}} \frac{\delta \Sigma}{\delta \Omega_{B \mu}{ }^{a}}+\frac{\delta \Sigma}{\delta \eta^{a}} \frac{\delta \Sigma}{\delta \Omega_{\eta}{ }^{a}}+b^{a} \frac{\delta \Sigma}{\delta \bar{c}^{a}}+\right. \\
\left.\quad+h^{a} \frac{\delta \Sigma}{\delta \bar{\phi}^{a}}+\frac{\delta \Sigma}{\delta c^{a}} \frac{\delta \Sigma}{\delta \Omega_{c}{ }^{a}}+\frac{\delta \Sigma}{\delta \phi^{a}} \frac{\delta \Sigma}{\delta \Omega_{\phi}{ }^{a}}\right), \\
\overline{\mathcal{G}}^{a}=\frac{\delta}{\delta \bar{c}^{a}}+\partial_{\mu} \frac{\delta}{\delta \Omega_{A \mu}} \\
\overline{\mathcal{F}}^{a}=\frac{\delta}{\delta \bar{\phi}^{a}}+\partial_{\mu} \frac{\delta}{\delta \Omega_{B \mu}} .
\end{aligned}
$$

In the Landau gauge the classical action is also invariant with respect to the following two integrated ghost equation:

$$
\begin{aligned}
\mathcal{G}^{a} \Sigma & =\Delta_{(g)}^{a}, \\
\mathcal{F}^{a} \Sigma & =\Delta_{(f)}^{a},
\end{aligned}
$$


where

$$
\begin{aligned}
\mathcal{G}^{a} & =\int \mathrm{d}^{3} x\left(\frac{\delta}{\delta c^{a}}+f^{a b c} \bar{c}^{b} \frac{\delta}{\delta b^{c}}+f^{a b c} \bar{\phi}^{b} \frac{\delta}{\delta h^{c}}\right), \\
\mathcal{F}^{a} & =\int \mathrm{d}^{3} x\left(\frac{\delta}{\delta \phi^{a}}+f^{a b c} \bar{\phi}^{b} \frac{\delta}{\delta b^{c}}\right), \\
\Delta_{(g)}^{a} & =\int \mathrm{d}^{3} x f^{a b c}\left(\Omega_{A \mu}{ }^{b} A_{\mu}^{c}+\Omega_{B \mu}^{b} B_{\mu}^{c}+\Omega_{\eta}^{b} \eta^{c}-\Omega_{c}^{b} c^{c}-\Omega_{\phi}^{b} \phi^{c}\right), \\
\Delta_{(f)}^{a} & =\int \mathrm{d}^{3} x\left\{f^{a b c}\left(+\Omega_{B \mu}^{b} A_{\mu}^{c}-\Omega_{\phi}^{b} c^{c}\right)+\Omega_{\eta}^{a}\right\} .
\end{aligned}
$$

By commuting these two operators with the Slavnov-Taylor identity we get two more rigid invariances:

$$
\begin{aligned}
& \mathcal{H}_{r i g}^{a} \Sigma=0, \\
& \mathcal{N}_{\text {rig }}^{a} \Sigma=0,
\end{aligned}
$$

where

$$
\begin{aligned}
& \mathcal{H}_{\text {rig }}^{a}=\int \mathrm{d}^{3} x \sum_{\varphi} f^{a b c} \varphi^{a} \frac{\delta}{\delta \varphi^{b}} \\
& \mathcal{N}_{\text {rig }}^{a}=\int \mathrm{d}^{3} x\left\{f^{a b c}\left(A_{\mu}^{b} \frac{\delta}{\delta B_{\mu}^{c}}+c^{b} \frac{\delta}{\delta \phi^{c}}+\bar{\phi}^{b} \frac{\delta}{\delta \bar{c}^{c}}+h^{b} \frac{\delta}{\delta b^{c}}+\Omega_{B_{\mu}^{b}}^{b} \frac{\delta}{\delta \Omega_{A_{\mu}^{c}}^{c}}+\Omega_{\phi}^{b} \frac{\delta}{\delta \Omega_{c}^{c}}\right)-\frac{\delta}{\delta \eta^{a}}\right\} .
\end{aligned}
$$

Now, if we define

$$
\begin{aligned}
& \hat{\Omega}_{A_{\mu}^{a}}^{a}=\Omega_{A_{\mu}^{a}}^{a}+\partial_{\mu} \bar{c}^{a} \\
& \hat{\Omega}_{B \mu}^{a}=\Omega_{B \mu}^{a}+\partial_{\mu} \bar{\phi}^{a}, \\
& \begin{aligned}
\hat{\Sigma}\left[A, B, c, \phi, \widehat{\Omega}_{A}, \hat{\Omega}_{B}, \Omega_{\eta}, \Omega_{c}, \Omega_{\phi}\right]= & \Sigma\left[A, B, c, \bar{c}, b, \phi, \bar{\phi}, h, \Omega_{A}, \Omega_{B}, \Omega_{\eta}, \Omega_{c}, \Omega_{\phi}\right]+ \\
& \quad-\left(b \wedge * \mathrm{~d}^{\dagger} A+h \wedge * \mathrm{~d}^{\dagger} B\right)
\end{aligned}
\end{aligned}
$$

the action $\widehat{\Sigma}$ satisfies the S.T. identity

$$
\widehat{B}_{\widehat{\Sigma}} \widehat{\Sigma}=0,
$$

where

$$
\begin{aligned}
& \widehat{B}_{\widehat{\Sigma}}=\int \mathrm{d}^{3} x\left(\frac{\delta \widehat{\Sigma}}{\delta A_{\mu}^{a}} \frac{\delta}{\delta \widehat{\Omega}_{A_{\mu}}^{a}}+\frac{\delta \widehat{\Sigma}}{\delta \Omega_{A_{\mu}}^{a}} \frac{\delta}{\delta A_{\mu}^{a}}+\frac{\delta \widehat{\Sigma}}{\delta B_{\mu}^{a}} \frac{\delta}{\delta \hat{\Omega}_{B_{\mu}}^{a}}+\frac{\delta \widehat{\Sigma}}{\delta \hat{\Omega}_{B_{\mu}^{a}}^{a}} \frac{\delta}{\delta B_{\mu}^{a}}+\frac{\delta \widehat{\Sigma}}{\delta \eta^{a}} \frac{\delta}{\delta \Omega_{\eta}{ }^{a}}+\frac{\delta \widehat{\Sigma}}{\delta \Omega_{\eta}{ }^{a}} \frac{\delta}{\delta \eta^{a}}+\right. \\
& \left.+\frac{\delta \widehat{\Sigma}}{\delta c^{a}} \frac{\delta}{\delta \Omega_{c}{ }^{a}}+\frac{\delta \widehat{\Sigma}}{\delta \Omega_{c}{ }^{a}} \frac{\delta}{\delta c^{a}}+\frac{\delta \hat{\Sigma}}{\delta \phi^{a}} \frac{\delta}{\delta \Omega_{\phi}{ }^{a}}+\frac{\delta \hat{\Sigma}}{\delta \Omega_{\phi}{ }^{a}} \frac{\delta}{\delta \phi^{a}}\right) .
\end{aligned}
$$


The Slavnov-Taylor operator $\widehat{B}_{\widehat{\Sigma}}$ is again nihilpotent and satisfies $\widehat{B}_{\gamma} \widehat{B}_{\gamma} \gamma=0 \forall \gamma$. The action of the Slavnov-Taylor operator on the fields and the sources is

$$
\begin{aligned}
B_{\widehat{\Sigma}} \varphi & =\frac{\delta \widehat{\Sigma}}{\delta \Omega_{\varphi}}=s \varphi \quad \text { for } \varphi=A, B, \eta, c, \phi, \\
B_{\widehat{\Sigma}} \widehat{\Omega}_{A \mu} & =\frac{\delta \Sigma}{\delta A_{\mu}}=i * \mathrm{~d}_{A} B-i g\left[\eta, B+\mathrm{d}_{A} \eta\right]+i g\left\{\widehat{\Omega}_{A}, c\right\}+i g\left\{\hat{\Omega}_{B}, \phi\right\}, \\
B_{\widehat{\Sigma}} \hat{\Omega}_{B \mu} & =\frac{\delta \widetilde{\Sigma}}{\delta B_{\mu}}=i * F+2\left(B+\mathrm{d}_{A} \eta\right)+i g\left\{\hat{\Omega}_{B}, c\right\}, \\
B_{\widehat{\Sigma}} \Omega_{\eta} & =\frac{\delta \widehat{\Sigma}}{\delta \eta}=-2 \mathrm{~d}_{A}\left(B+\mathrm{d}_{A} \eta\right)-i g\left[\Omega_{\eta}, c\right], \\
B_{\widehat{\Sigma}} \Omega_{c} & =\frac{\delta \widehat{\Sigma}}{\delta c}=-\mathrm{d}_{A}^{\dagger} \widehat{\Omega}_{A}+i g *\left[\hat{\Omega}_{B}, * B\right]+i g\left[\Omega_{\phi}, \eta\right]+i g\left[\Omega_{c}, c\right]+i g\left[\Omega_{\eta}, \phi\right], \\
B_{\widehat{\Sigma}} \Omega_{\phi} & =\frac{\delta \widehat{\Sigma}}{\delta \phi}=-\mathrm{d}_{A}^{\dagger} \hat{\Omega}_{B}+\Omega_{\eta}+i g\left[\Omega_{\phi}, c\right] .
\end{aligned}
$$

\subsection{Anomaly}

The constraints 4 4.2, 1.3, 4.8) renormalize as in the gaussian formulation, so that we can think the action functional $\Gamma$ as satisfying the following constraints:

$$
\begin{aligned}
\frac{\delta}{\delta b^{a}} \Gamma & =\frac{\delta}{\delta h^{a}} \Gamma=0, \\
\overline{\mathcal{G}}^{a} \Gamma & =\overline{\mathcal{F}}^{a} \Gamma=0, \\
\mathcal{G}^{a} \Gamma & =\Delta_{(g)}^{a}, \\
\mathcal{F}^{a} \Gamma & =\Delta_{(f)}^{a}, \\
\mathcal{H}_{\text {rig }}^{a} \Gamma & =0, \\
\mathcal{N}_{\text {rig }}^{a} \Gamma & =0 .
\end{aligned}
$$

Then it can be decomposed in the same way as the classical action $\Sigma$ :

$$
\begin{aligned}
\Gamma\left[A, B, c, \bar{c}, b, \phi, \bar{\phi}, h, \Omega_{A}, \Omega_{B}, \Omega_{\eta}, \Omega_{c}, \Omega_{\phi}\right]= & \widehat{\Gamma}\left[A, B, c, \phi, \widehat{\Omega}_{A}, \hat{\Omega}_{B}, \Omega_{\eta}, \Omega_{c}, \Omega_{\phi}\right]+ \\
& +\left(b \wedge * \mathrm{~d}^{\dagger} A+h \wedge * \mathrm{~d}^{\dagger} B\right) .
\end{aligned}
$$

For what concerns the renormalization of the Slavnov-Taylor identity, we follow the strategy outlined in the previous section. Therefore we assume that there is no breaking till the order $(n-1)$ and study the next order:

$$
\widehat{B}_{\widehat{\Gamma}} \widehat{\Gamma}=\hbar^{n} \Delta+O\left(\hbar^{n+1}\right),
$$

where $\Delta$ is a functional of dimensions $d_{U V} \leq \frac{7}{2}$ and $d_{I R} \geq 3$ by virtue of the QAP, and satisfies the constraints (4.17,4.18,4.21, 4.22 ) and

$$
\begin{aligned}
\mathcal{G}^{a} \Delta & =0, \\
\mathcal{F}^{a} \Delta & =0
\end{aligned}
$$

The nihilpotency properties of the Slavnov-Taylor operators imply the consistency condition

$$
\widehat{B}_{\widehat{\Sigma}} \Delta=0 .
$$


To solve (4.25) we consider the linearized problem

$$
b_{0} \Delta=0
$$

where $b_{0}$ is the linear part of $\widehat{B}_{\widehat{\Sigma}}$ and satisfies $b_{0}^{2}=0$. Because the fields $\eta$ and $\phi$ are a $b_{0}$-doublet, then the cohomology of $b_{0}$ does not depend on them; moreover the proposition (3.1) still holds.

Therefore we can use the results of the previous section and conclude that also this formulation of the BFYM theory is not anomalous.

\subsection{Stability}

Now, to study the most general invariant counterterms we have to solve

$$
B_{\Sigma} L=0
$$

in the space of local field functionals of ghost number zero and dimension $d_{U V} \leq 3$ and $d_{I R} \geq 3$, that satisfy the previous constraints; the ghost equations are written in this case as

$$
\begin{aligned}
\mathcal{G}^{a} L & =0 \\
\mathcal{F}^{a} L & =0
\end{aligned}
$$

Due to the gauge conditions and the antighost equations, equation (4.27) reduces to

$$
\widehat{B}_{\widehat{\Sigma}} L=0
$$

Solving the linearized equation

$$
b_{0} L=0,
$$

with the aid of proposition (3.1) we find

$$
\Omega_{3}^{0}=b_{0} \Omega_{3}^{-1}+\mathrm{d} \Omega_{2}^{0}+u A \mathrm{~d} A+v B \mathrm{~d} A+z \mathrm{~d} A * \mathrm{~d} A .
$$

But the term of coefficient $v$ is equivalent to $F * F$, indeed

$$
B \mathrm{~d} A=-\frac{1}{2} i \mathrm{~d}_{A} * \mathrm{~d}_{A}+\frac{1}{2} b_{0}\left(\Omega_{B} \mathrm{~d} A\right)-d(\eta \mathrm{d} A),
$$

therefore equation (4.32) becomes

$$
\Omega_{3}^{0}=b_{0} \Omega_{3}^{-1}+\mathrm{d} \Omega_{2}^{0}+z_{1} \mathrm{~d} A * \mathrm{~d} A+z_{2} A \mathrm{~d} A .
$$

The extension to the cohomology of the S.T. operator is straightforward:

$$
\Omega_{3}^{0}=\widehat{B}_{\widehat{\Sigma}} \Omega_{3}^{-1}+\mathrm{d} \Omega_{2}^{0}+z_{1} F * F+z_{2} \mathcal{L}_{\mathrm{CS}} .
$$

The trivial part is given by

$$
\begin{aligned}
& \widehat{B}_{\widehat{\Sigma}}\left[t_{1} \widehat{\Omega}_{A} * A+t_{2} \hat{\Omega}_{B} * B+t_{3} \Omega_{\eta} * \eta+t_{4} \Omega_{c} * c+t_{5} \Omega_{\phi} * \phi+t_{6} \hat{\Omega}_{B} \mathrm{~d} A+t_{7} \hat{\Omega}_{B} A A+\right. \\
& \left.\quad+t_{8} \hat{\Omega}_{B} * \mathrm{~d} \eta+t_{9} \hat{\Omega}_{B} * A \eta+t_{10}\left(\hat{\Omega}_{B} * A \eta^{2}+\text { perm. }\right)+t_{11}\left(\hat{\Omega}_{B} A A \eta+\text { perm. }\right)\right]
\end{aligned}
$$


and it is easily seen that the topological rigid invariance (4.22) implies that

$$
\begin{aligned}
& t_{1}=-t_{2}, \\
& t_{3}=t_{5}=t_{9}=t_{10}=t_{11}=0 ;
\end{aligned}
$$

in this way we get rid of all the non parity invariant trivial counterterms. Moreover the ghost equation (4.28) implies that

$$
t_{4}=0
$$

Explicitly we see that

$$
\begin{aligned}
& \widehat{B}_{\widehat{\Sigma}}\left(\widehat{\Omega}_{A} * A\right)=\left(N_{A}-N_{\widehat{\Omega}_{A}}\right) \widehat{\Sigma}=\mathcal{N}_{A} \widehat{\Sigma}, \\
& \widehat{B}_{\widehat{\Sigma}}\left(\hat{\Omega}_{B} * B\right)=\left(N_{B}-N_{\hat{\Omega}_{B}}\right) \widehat{\Sigma}=\mathcal{N}_{B} \widehat{\Sigma}, \\
& \widehat{B}_{\widehat{\Sigma}}\left(\hat{\Omega}_{B} \mathrm{~d} A\right)=\left(N_{B \rightarrow * \mathrm{~d} A}-N_{\widehat{\Omega}_{A} \rightarrow * \mathrm{~d} \hat{\Omega}_{B}}\right) \widehat{\Sigma}=\mathcal{N}_{\text {rot }}^{(1)} \widehat{\Sigma}, \\
& \widehat{B}_{\widehat{\Sigma}}\left(\hat{\Omega}_{B}[A, A]\right)=\left(N_{B \rightarrow *[A, A]}-2 N_{\widehat{\Omega}_{A} \rightarrow *\left[A, \hat{\Omega}_{B}\right]}\right) \widehat{\Sigma}=\mathcal{N}_{\text {rot }}^{(2)} \widehat{\Sigma}, \\
& \widehat{B}_{\widehat{\Sigma}}\left(\hat{\Omega}_{B} * \mathrm{~d} \eta\right)=\left(N_{B \rightarrow \mathrm{d} \eta}-N_{\Omega_{\eta} \rightarrow \mathrm{d}^{\dagger} \hat{\Omega}_{B}}\right) \widehat{\Sigma}=\mathcal{N}_{\text {rot }}^{(3)} \widehat{\Sigma} .
\end{aligned}
$$

We have thus found that the most general invariant action giving $\widehat{\Sigma}$ in the $\hbar \rightarrow 0$ limit is given by

$$
\begin{aligned}
\widehat{\Sigma} & +\hbar^{n} z_{1} \operatorname{Tr} \int F_{A} * F_{A}+i \hbar^{n} z_{2} m S_{C S}+ \\
& +\hbar^{n} a_{1}\left(\mathcal{N}_{A}-\mathcal{N}_{B}\right) \widehat{\Sigma}+\hbar^{n} \frac{a_{2}}{g^{2}} \mathcal{N}_{\text {rot }}^{(1)} \widehat{\Sigma}+\hbar^{n} \frac{a_{3}}{g^{2}} \mathcal{N}_{\text {rot }}^{(2)} \widehat{\Sigma}+\hbar^{n} a_{4} \mathcal{N}_{\text {rot }}^{(3)} \widehat{\Sigma}
\end{aligned}
$$

\subsection{Renormalization transformations}

With a transformation of the fields similar to that for the gaussian formulation we can absorb all the counterterms that we found:

$$
\begin{aligned}
A_{0} & =A_{R}-\hbar^{n} a_{1} A_{R}, \\
B_{0} & =B_{R}+\hbar^{n}\left(a_{1}-z_{1}\right) B_{R}+\hbar^{n} \frac{1}{g^{2}}\left(-a_{2}+i z_{1}\right) * \mathrm{~d} A_{R}-\frac{i}{2} \hbar^{n} \frac{1}{g^{2}}\left(-2 a_{3}+i z_{1}\right) *\left[A_{R}, A_{R}\right]-\hbar^{n} a_{4} \mathrm{~d} \eta_{R}, \\
\eta & =\eta_{R}-\hbar^{n} z_{1} \eta_{R}, \\
c_{0} & =c_{R} \\
\phi_{0} & =\phi_{R}-\hbar^{n} z_{1} \phi_{R}, \\
\widehat{\Omega}_{A 0} & =\widehat{\Omega}_{A R}+\hbar^{n} a_{1} \widehat{\Omega}_{A R}+\hbar^{n} \frac{1}{g^{2}}\left(a_{2}-i z_{1}\right) * \mathrm{~d} \hat{\Omega}_{B R}-i \hbar^{n} \frac{1}{g^{2}}\left(2 a_{3}-i z_{1}\right) *\left[A_{R}, \hat{\Omega}_{B R}\right], \\
\hat{\Omega}_{B 0} & =\hat{\Omega}_{B R}+\hbar^{n}\left(-a_{1}+z_{1}\right) \hat{\Omega}_{B R}, \\
\Omega_{\eta_{0}} & =\Omega_{\eta_{R}}+\hbar^{n} z_{1} \Omega_{\eta_{R}}+\hbar^{n} a_{4} \mathrm{~d}^{\dagger} \Omega_{B R}, \\
\Omega_{c 0} & =\Omega_{c R}, \\
\Omega_{\phi_{0}} & =\Omega_{\phi_{R}}+\hbar^{n} z_{1} \Omega_{\phi_{R}}, \\
g_{0} & =g_{R}+\hbar^{n} z_{1} g_{R}, \\
m_{0} & =m_{R}-\hbar^{n} z_{2} m_{R} .
\end{aligned}
$$

We then conclude that the theory is algebraically stable, and again note that only the $F^{2}$ term contributes to the physical renormalization of $g$. 


\section{Conclusions}

In this paper we have considered the first order BF formulation of 3D YM theory. Two different models have been introduced, named gaussian BFYM and extended BFYM, with

a different symmetry and field contents but both classically equivalent to the standard YM theory.

We have quantized the models, introduced a Chern-Simons IR regularization mass and discussed their renormalization properties. In particular, using algebraic tools, we have shown that both the models are anomaly free and stable against radiative corrections; the physical renormalizations of the coupling and of the CS mass occurr exactly as in the standard YMCS case. Moreover we have given a detailed analysis of the renormalization tranformations which produce all the invariant counterterms required.

\section{Acknowledgments}

The authors acknowledge useful discussions with A. Grassi, A.A. acknowledges some remarks by O. Piguet. This work has been partially supported by MURST and by TMR programme ERB-4061-PL-95-0789 in which M.Z. is associated to Milan. 


\section{A Feynman rules}

\section{A.1 Gaussian model}

The propagator matrix for the gaussian model is

$$
\Delta^{a b}(x-y)=\left(\begin{array}{ccc}
\Delta_{A A^{a b}}(x-y) & \Delta_{A B}{ }^{a b}(x-y) & \Delta_{A b}{ }^{a b}(x-y) \\
\Delta_{B A^{a b}}(x-y) & \Delta_{B B}{ }^{a b}(x-y) & \Delta_{B b}{ }^{a b}(x-y) \\
\Delta_{b A}{ }^{a b}(x-y) & \Delta_{b B}{ }^{a b}(x-y) & \Delta_{b b}{ }^{a b}(x-y)
\end{array}\right)
$$

i.e.

$$
\Delta^{a b}(p)=\left(\begin{array}{ccc}
\frac{1}{p^{2}} P_{\mu \nu}-\alpha \frac{p_{\mu} p_{\nu}}{p^{4}} & -\varepsilon_{\mu \rho \nu} \frac{p_{\rho}}{p^{2}} & i \frac{p_{\mu}}{p^{2}} \\
-\epsilon_{\mu \rho \nu} \frac{p_{\rho}}{p^{2}} & \frac{p_{\mu} p_{\nu}}{p^{2}} & 0 \\
-i p_{\nu} p^{2} & 0 & 0
\end{array}\right) \delta^{a b}
$$

where $P_{\mu \nu}=\delta_{\mu \nu}-\frac{p_{\mu} p_{\nu}}{p^{2}}$. BFYM theory has only the vertex $B A A$ and the ghost one, and indeed the off diagonal structure of the propagator matrix is relevant in recovering the non linear self interactions of YM theory.

Note that the propagator $\Delta_{B B}$ in (A.2) is not transversal; a closer analysis of it reveals some problems. Indeed if we calculate 1-loop correction for this propagator it appears to have a transverse structure of the type $P_{\mu \nu}$. This structure agrees with the Ward identity for the quantum propagator $G_{B B}$, which requires to all orders

$$
p_{\mu} G_{B_{\mu} B_{\nu}}=0
$$

This mismatch can be explained observing that in the inversion of the kinetic term we have used the naive measure over $B$, while configurations of the type $B=\mathrm{d}_{A} \xi$, which are non dynamical owing to the Bianchi identity (they do not couple in the term $B \wedge F$ ), give a spurious contribution to $\Delta_{B B}$ which has to be subtracted.

This fact is better understood considering the equivalence between equations (2.7) and (2.12) and choosing $\mathrm{d}_{A}^{\dagger} B=0$ as the topological gauge-fixing condition to use in (2.12). The functional measure becomes

$$
\begin{aligned}
& \mathcal{D} B \delta\left(\mathrm{d}_{A}^{\dagger} B\right) \mathcal{D} \eta \operatorname{det}\left(\mathrm{d}_{A}^{\dagger} \mathrm{d}_{A}\right) e^{-g^{2} \operatorname{Tr}\left(2 B \wedge * \mathrm{~d}_{A} \eta+\mathrm{d}_{A} \eta \wedge * \mathrm{~d}_{A} \eta\right)}= \\
& \mathcal{D} B \mathcal{D} \eta \delta\left(B-B_{0}\right) \frac{1}{\operatorname{det}\left(\mathrm{d}_{A}^{\dagger} \mathrm{d}_{A}\right)^{\frac{1}{2}}} \operatorname{det}\left(\mathrm{d}_{A}^{\dagger} \mathrm{d}_{A}\right) e^{-g^{2} \operatorname{Tr}\left(2 B \wedge * \mathrm{~d}_{A} \eta+\mathrm{d}_{A} \eta \wedge * \mathrm{~d}_{A} \eta\right)}
\end{aligned}
$$

where $B_{0}$ are the configurations such that $\mathrm{d}_{A}^{\dagger} B_{0}=0$. After the $\eta$ integration, which gives $\left(\operatorname{det} \mathrm{d}_{A}^{\dagger} \mathrm{d}_{A}\right)^{-\frac{1}{2}}$, the measure reads

$$
\mathcal{D} B \delta\left(B-B_{0}\right)
$$

which means that the extended formulation is equivalent to exclude from the functional integration the "longitudinal" B-fields. Therefore these degrees of freedom have to be 
disposed of in the gaussian formulation. Then the correct propagator $\Delta_{B B}$ turns out to be

$$
\Delta_{B_{\mu} B_{\nu}}=-\left(\delta_{\mu \nu}-\frac{p_{\mu} p_{\nu}}{p^{2}}\right)
$$

that satisfies the identity (A.3). In conclusion we have the following Feynman rules:

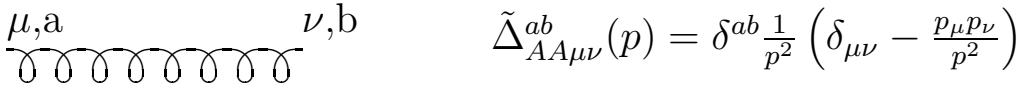

$$
\begin{aligned}
& p \longrightarrow \\
& \gamma_{\gamma \gamma \mathrm{a}}^{\mu, \mathrm{b}} \quad \tilde{\Delta}_{B A \mu \nu}^{a b}(p)=-\varepsilon_{\mu \rho \nu} \delta^{a b} \frac{p_{\rho}}{p^{2}} \\
& p \longrightarrow \\
& \frac{\mu, \mathrm{a} \quad \nu, \mathrm{b}}{p \longrightarrow} \quad \tilde{\Delta}_{B B \mu \nu}^{a b}(p)=-\left(\delta_{\mu \nu}-\frac{p_{\mu} p_{\nu}}{p^{2}}\right) \\
& \frac{\mathrm{a}}{-}-\underset{\bar{p}}{\leftarrow}-\mathrm{b} \quad \tilde{\Delta}_{\bar{c} c}^{a b}(p)=-\delta^{a b} \frac{1}{p^{2}} \\
& \rho^{\rho, a} \tilde{\Lambda}_{(B A A) \mu \nu \rho}^{a b c}=-i g f^{a b c} \varepsilon_{\mu \nu \rho} \\
& 2 \nu, b
\end{aligned}
$$

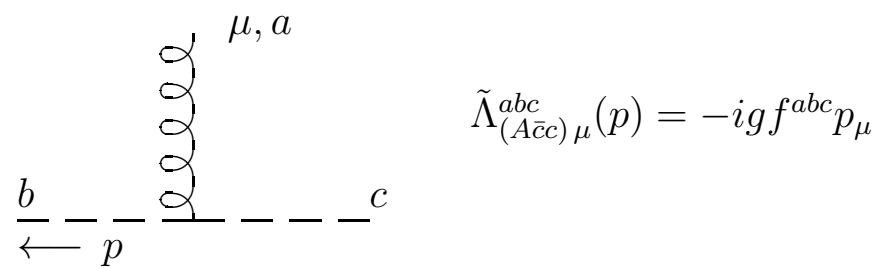

\section{A.2 Extended formulation}

In this case the propagator matrix becomes

$$
\left(\begin{array}{ccccc}
\frac{1}{p^{2}} P_{\mu \nu}-\alpha \frac{p_{\mu} p_{\nu}}{p^{4}} & -\epsilon_{\mu \nu \rho \frac{p_{\rho}}{p^{2}}} & 0 & i \frac{p_{\mu}}{p^{2}} & 0 \\
-\epsilon_{\mu \nu \rho \frac{p_{\rho}}{p^{2}}} & -\beta \frac{p_{\mu} p_{\nu}}{p^{4}} & -i \beta \frac{p_{\mu}}{p^{4}} & 0 & i \frac{p_{\mu}}{p^{2}} \\
0 & i \beta \frac{p_{\mu}}{p^{4}} & \frac{1}{p^{2}}-\beta \frac{1}{p^{4}} & 0 & \frac{1}{p^{2}} \\
-i \frac{p_{\nu}}{p^{2}} & 0 & 0 & 0 & 0 \\
0 & -i \frac{p_{\nu}}{p^{2}} & \frac{1}{p^{2}} & 0 & 0
\end{array}\right) .
$$

We observe that in this formulation of the theory the propagators satisfy the Ward identities because the introduction of the $\eta$-field and the topological gauge-fixing separate the transverse and longitudinal parts of the $B$-field. 


\section{B Ward identities on two point functions}

In this appendix we collect the Ward identities on two point functions for the gaussian model. Organizing the propagator matrix of the fields $A$ and $B$ as

$$
\Delta=\left(\begin{array}{cc}
\Delta_{A A} & \Delta_{A B} \\
\Delta_{B A} & \Delta_{B B}
\end{array}\right),
$$

we have with similar notation

$$
\begin{aligned}
& \partial_{\mu} \partial_{\nu} \Delta_{\mu \nu}^{a b}(x-y)=\left(\begin{array}{ll}
\alpha & 0 \\
0 & 0
\end{array}\right) \delta^{a b} \delta^{(3)}(x-y), \\
& \partial_{\mu} \partial_{\nu} G_{\mu \nu}^{a b}(x-y)=\left(\begin{array}{ll}
\alpha & 0 \\
0 & 0
\end{array}\right) \delta^{a b} \delta^{(3)}(x-y), \\
& \partial_{\mu} \partial_{\nu} \Gamma_{\mu \nu}^{a b}(x-y)=\left(\begin{array}{cc}
0 & 0 \\
0 & \gamma\left(x-y, g^{2}\right) \partial^{2}
\end{array}\right) \delta^{a b} \delta^{(3)}(x-y), \\
& \partial_{\mu} \partial_{\nu} \Sigma_{\mu \nu}^{a b}(x-y)=\left(\begin{array}{cc}
0 & 0 \\
0 & \left(1-\gamma\left(x-y, g^{2}\right)\right) \partial^{2}
\end{array}\right) \delta^{a b} \delta^{(3)}(x-y),
\end{aligned}
$$

for the complete propagators $G$, for the inverse quantum propagators $\Gamma$ and for the selfenergies $\Sigma$.

\section{Notations and conventions}

In the paper we have used the following conventions

$$
\operatorname{Tr}\left(T^{a} T^{b}\right)=\frac{1}{2} \delta^{a b} \quad\left[T^{a}, T^{b}\right]=i f^{a b c} T^{c}
$$

where the $T$ 's are the generators of a representation of the Lie algebra of the gauge group and $f^{a b c}$ the structure constants.

The covariant derivative is

$$
\mathrm{d}_{A}=\mathrm{d}-i[A, \cdot]
$$

and the Hodge-adjoint operators are defined as

$$
\begin{aligned}
\mathrm{d}^{\dagger} & =* \mathrm{~d} * \\
\mathrm{~d}_{A}^{\dagger} & =* \mathrm{~d}_{A} *
\end{aligned}
$$

where $*$ is the Hodge duality operator. We also write $F=F_{\mu \nu} d x^{\mu} \wedge d x^{\nu}$.

The inner product in the space of Lie algebra valued q-forms is

$$
(\varphi, \omega)=\int \operatorname{Tr}(\varphi \wedge * \omega),
$$

which in the euclidean space is positive-definite. We have also used $\varphi^{2}=(\varphi, \varphi)$. 


\section{References}

[1] E. Witten, Commun. Math. Phys. 117(1988)353-386

[2] G. T. Horowitz, Commun. Math. Phys. 125(1989)417-436

[3] M. Blau, G. Thompson, Ann. Phys (NY) 205 (1991) 130-172

[4] G. T. Horowitz and M. Srednicki, Commun. Math. Phys. 130(1990)83

[5] F. Fucito, M. Martellini, M. Zeni, The BF formalism for QCD and quark confinement, hep-th 9605018.

[6] M. Martellini, M. Zeni, Feynman rules and $\beta$-function for the BF Yang-Mills theory, hep-th 9702035, to be published in Phys. Lett. B.

[7] A.S. Cattaneo, P. Cotta-Ramusino, F. Fucito, M. Martellini, A. Tanzini, M. Zeni, The BF topological embedding and non-Abelian pure gauge theories, IFUM 549/FT, $\mathrm{ROM} 2 \mathrm{~F} / 96 / 61$.

[8] A.S. Cattaneo, P. Cotta-Ramusino, A. Gamba, M. Martellini, Phys. Lett. B355(1995)245

[9] G. 't Hooft, Nucl. Phys. B138(1978)1, Nucl. Phys. 153(1979)141

[10] N. Maggiore, S.P. Sorella, Nucl. Phys. B377 (1992) 236-251

[11] A. S. Schwarz, Commun. Math. Phys. 67 (1979) 1

[12] D. Birmingham, M. Blau, M. Rakowsky, G. Thompson, Phys. Rep. 209(1991)129340

[13] S. Deser, R. Jackiw, Phys. Rev. D23(1981)2291-2304

[14] G. 't Hooft, Acta Phys. Austr. Suppl. XXII(1980)531-586

[15] J.F. Schonfeld, Nucl. Phys. B185(1981)157-171

[16] S. Deser, R. Jackiw, S. Templeton, Phys. Rev. Lett. 48(1981)975-978

S. Deser, R. Jackiw, S. Templeton, Ann. Phys. (NY) 140(1982)372-411 e ERRATum Ann. Phys. (NY) 185(1988)406

[17] J.H. Lowenstein, Phys. Rev. D4(1971)2281-2290

Y.M.P. Lam, Phys. Rev. D6(1972)21452167

Y.M.P. Lam, Phys. Rev. D7(1973)2943-2949

T.E. Clark, J.H. Lowenstein, Nucl. Phys. B113(1976)109-134

[18] O. Piguet, S. P. Sorella, "Algebraic Renormalization", Lecture Notes in Physics m.28, Springer 1995

[19] C. Becchi, A. Rouet and R. Stora, Phys. Lett. 52B(1974)344; Commun. Math. Phys. 42(1975)127.

[20] R.D. Pisarski, S. Rao, Phys. Rev. D32(1985)2081-2096

[21] G. Giavarini, C.P. Martin, F. Ruiz Ruiz, Nucl. Phys. B381(1992)222-280

[22] A. Blasi, O. Piguet, S.P. Sorella, Nucl. Phys. B356(1991)154-162 